\title{
Systemic treatment options for advanced biliary tract carcinoma
}

\author{
Changqing Xie $^{1}\left[\right.$ Dicole A. McGrath ${ }^{1} \cdot$ Cecilia Monge Bonilla $^{1} \cdot$ Jianyang Fu $^{1}$
}

Received: 19 May 2020/Accepted: 25 July 2020/Published online: 3 August 2020

(C) The Author(s) 2020

\begin{abstract}
Advanced biliary tract cancers (BTC) include a diverse collection of rare and heterogenous tumors with poor prognosis. The combination of gemcitabine and cisplatin is the established first-line therapy for advanced BTC. There are no accepted standard treatments in the second line setting, though there are several ongoing clinical trials that implement chemotherapy as a therapeutic strategy. The understanding of the molecular landscape of BTC has offered hope of targeted therapies to the identified actionable genomic aberrations, such as FGFR2 gene fusions, mutations of IDH1/2, HER2, BRAC1/2 and BRAF. Pembigatinib has become the first approved targeted therapy for BTC with FGFR2 fusion or other rearrangements. Recent immunotherapy has opened new therapy avenues in BTC with pembrolizumab approved for either microsatellite instability high (MSI-H) or DNA mismatch repair deficient (dMMR) advanced solid tumors, including BTC. The combination of immunotherapy with other modalities is currently being evaluated in different clinical trials, since single agent immunotherapy appears to provide modest benefits in advanced BTC. In this review, we summarize the current status of treatment options, including systemic chemotherapy, targeted therapy, immunotherapy, and various combinations in advanced BTC.
\end{abstract}

Keywords Biliary tract cancer - Chemotherapy - Targeted therapy $\cdot$ Immunotherapy

Changqing Xie

changqing.xie@nih.gov

1 Thoracic and Gastrointestinal Malignancies Branch, Center for Cancer Research, National Cancer Institute, National Institutes of Health, Bethesda, MD, USA

\section{Introduction}

Biliary tract carcinoma (BTC) typically refers to malignancies originating in the intra- and extrahepatic biliary ductal system, known as cholangiocarcinoma (CCA), and the gallbladder; however, periampullary tumors are also included as BTCs. The primary risk factors for intrahepatic cholangiocarcinoma (iCCA) and extrahepatic cholangiocarcinoma (eCCA) include chronic inflammatory diseases of the biliary tract such as primary sclerosing cholangitis and hepatolithiasis, congenital hepatobiliary anomalies, liver fluke infection, non-alcoholic fatty liver disease [1-4]. Moreover, cholelithiasis with the presence of chronic inflammation is the most prevalent risk factor for gallbladder cancer (GBC) [5]. The anatomic subtypes of BTC display distinguished molecular aberrations [6], that suggests complexities of pathogenesis of BTC. Commonly occurring genetic aberrations in iCCA include TP53 (35\%), KRAS (24\%), ARID1A (20\%), IDH1 (18\%), MCL1 (16\%), and PBRM1 (11\%). In contrast, the most frequent aberrations in eCCA are TP53 (45\%), KRAS (40\%), ERBB2 (25\%), SMAD4 (25\%), FBXW7 (15\%) and CDKN2A (15\%) [7]. The clinical presentation of BTC differs with their sites, that patients with eCCA usually present with symptoms and painless jaundice is the most common, while the most common presentation of iCCA and GBC is of incidental discovery [1].

Although BTC is a relatively uncommon diagnosis, studies have suggested that the incidence and mortality of BTC has increased in recent years [8-10]. Among new cases of BTC that are diagnosed each year in the United States, there are approximately 3,000 cases of intrahepatic cholangiocarcinoma (iCCA), 11,000 cases of extrahepatic cholangiocarcinoma (eCCA) and gallbladder carcinoma 
(GBC) [11]. Possibly due to these location related mutations and the ease of total resection, patients diagnosed with distal eCCA tend to have better outcomes than patients with perihilar eCCA and iCCA [12]. The current 5-year survival rate in patients with early stage BTC who undergo curative-intent surgery is $30 \%$ [13]. Patients with advanced disease at diagnosis have limited treatment options and poor prognosis. This review is focused on systemic therapy options for advanced BTC, including chemotherapy, targeted therapy, and immunotherapy.

\section{Chemotherapy}

Systemic chemotherapy is the standard of care for the patients with advanced BTC. There is evidence from several trials to support the use of gemcitabine-based or fluoropyrimidine-based chemotherapy for patients with advanced BTC [14-17]. Among these, a randomized, multicenter, phase III study in advanced BTC evaluated overall survival (OS) with the combination of gemcitabine/cisplatin (GemCis) in comparison to gemcitabine alone (ABC-02) [18]. In total, 410 patients with locally advanced and metastatic BTC were enrolled and randomly assigned to a treatment. The results showed that the addition of cisplatin significantly improved progression-free survival (PFS) and OS by 3 months. The median PFS and OS were 8 and 11.7 months, respectively, with a median follow-up period of 8.2 months in the GemCis cohort. The tumor control rate was $81.4 \%$ in the GemCis cohort, compared to $71.8 \%$ in treatment with gemcitabine alone [18]. This study established GemCis as the current first line of care chemotherapy in advanced BTC, with subsequent studies further validating their results.

For example, a similar regimen was tested in a multicenter, randomized, phase II study of advanced BTC in Japan (BT22). Among 83 patients analyzed, the overall response rate (ORR) was $19.5 \%$ in the GemCis combination cohort versus $11.9 \%$ in the gemcitabine treatment group. Adding cisplatin to the treatment regimen increased OS and PFS by 2 and 3 months, respectively [16]. Overall, the combination was safe and adverse events were similar in the two groups, with the exception of a higher incidence of neutropenia in the GemCis group [16, 18]. Subsequent additions to gemcitabine/platinum with targeted therapeutic agents have failed to demonstrate better outcomes to date, with a range of median OS of 7.7-14.1 months [17, 19-21]. A recent report from a phase III randomized trial have posited that the combination of gemcitabine plus oral fluoropyrimidine S-1 showed non-inferior to GemCis in terms of OS and PFS in comparison to GemCis regimen and well tolerated among recruited 354 patients, although there were schedule difference of GemCis used in this study and those in the original ABC studies [22]. There are ongoing trials utilizing the strategies with combinations of targeted therapy and chemotherapy with the hope of new first line of care options for patients with advanced unresectable BTC (Table 1). Unfortunately, no randomized phase III trials with such combinations are yet in progress.

More intensive, triple chemotherapy regimens have been evaluated and have proven to be effective in a small number of patients with advanced BTC. A randomized phase III trial was conducted to evaluate the combination of epirubicin, cisplatin and 5FU (ECF) versus 5FU, etoposide and leucovorin (FELV) in patients with advanced BTC as a first line of treatment. The results from enrollment of 54 patients showed no difference between two groups in terms of ORR and median OS for ECF and FELV were 9.02 months and 12.03 months, respectively; however, no significant difference was detected between the groups [23]. In a more recent phase II trial of advanced BTC, patients were treated with the addition of 5-FU to GemCis (GFP). There were 21 patients enrolled and impressive anti-tumoral activity was exhibited by a median OS of 18.8 months and a median time to progression of 13.4 months [24]. Additionally, fifty patients with advanced BTC in the KHBO 1002 phase II trial were treated with GemCis/S-1 and showed a median OS of 16.1 months [25]. The phase III trial of this combination has been completed and authors reported similar findings to their phase II trial (KHBO1401-MITSUBA) [26]. Finally, the combination of GemCis/nab-paclitaxel was tested in a phase II trial and 60 patients with metastatic or unresectable BTC showed a median PFS of 11.4 months and median OS of 19.2 months [27].

There is no recommendation for a second-line therapy; however, numerous of trials with various chemotherapy combinations have been conducted on the subject. A systematic review of second-line chemotherapies in advanced BTC has been conducted, which included 25 studies (14 phase II clinical trials, 9 retrospective analyses, and 2 case reports) and the outcomes from 761 patients were reported. The mean PFS, OS, and response rate were 3.2 months, 7.2 months, and 7.7\%, respectively [28]. ABC-06 is an open-label, randomized, multicenter study comparing FOLFOX (5-FU and oxaliplatin) as second line option with active symptom control after GemCis, that was recently completed (NCT01926236). The updated results showed that 81 patients in the FOLFOX cohort had a median OS of 6.2 months compared to 5.3 months in the cohort with active symptom control alone [29]. Though all of the trials had few patients, median OS ranged from 4 to 9 months, suggesting that treatment in the refractory setting is feasible and may provide a survival advantage when compared to active symptom control. Ongoing clinical trials utilizing chemotherapy to treat BTC are outlined in Table 1. 
Table 1 Ongoing trials of chemotherapy with or without targeted therapy in advanced BTC

\begin{tabular}{llll}
\hline Regimen & Phase & Trial ID & Design \\
\hline Trifluridine/Tipiracil/Irinotecan & II & NCT04059562 & AC \\
Trifluridine/Tipiracil & II & NCT04076761 & AC \\
FOLFIRINOX & II & NCT04305288 & AC \\
FORFIRINOX & II & NCT03772132 & BD \\
Nal-IRI/5-FU/leucovorin & II & NCT03043547 & AC \\
Irinotecan/Trifluridine and Tipiracil & II & NCT04072445 & AC \\
Nal-Irinotecan/Trifluridine and Tipiracil & I/II & NCT03368963 & AC \\
Chemotherapy/EphB4-HAS/ & I & NCT02495896 & AC \\
GemCis + Copanlisib & II & NCT02631590 & AC \\
GemCis/Anetumab ravtansine & I & NCT03102320 & BD \\
FORFIRINOX/target agents & II & NCT03768375 & BD \\
GEMOX/target agents & II & NCT02836847 & BD \\
GemCis/Ivosidenib/Pemigatinib & I & NCT04088188 & BD \\
GemCis/CPI 613 & I/II & NCT04203160 & AC \\
GemCis/CX-4945 & I/II & NCT02128282 & AC \\
GemCis/Pemigatinib & I/II & NCT02393248 & BD \\
Carboplatin/Paclitaxel/Pevonedistat & II & NCT04175912 & AC \\
GemCis/FT-2102 & I/II & NCT03684811 & BD \\
\hline
\end{tabular}

FORFIRINOX folinic acid, fluorouracil, irinotecan, oxaliplatin, nal-IRI liposomal irinotecan, GemCis gemcitabine cisplatin, GEMOX gemcitabine oxaliplatin, $A C$ all-comers, $B D$ biomarker driven

\section{Targeted therapies}

The genetic landscape of BTC has been illuminated with the widespread use of next generation sequencing. More importantly, the understanding of this landscape has led to the identification of novel actionable drivers of BTC pathogenesis $[3,30]$ and rapid clinical trial development targeting various molecular aberrations (Table 2).

\section{FGFR inhibitors}

Fibroblast growth factor receptor (FGFR) genetic alterations (fusions, amplifications, mutations) occur in 7-45\% of iCCA cases, $<1-5 \%$ eCCA cases, and 3\% GBC cases [30, 31]. Currently, many active clinical trials are enrolling patients to further evaluate the role of FGFR2 inhibitors in the management of BTC.

\section{Selective inhibitor}

Pemigatinib (INCB054828) is a selective, oral inhibitor of FGFR 1, 2 and 3. A multicenter, open-label, single-arm, multicohort, phase II (FIGHT-202) study showed an ORR of $35.5 \%$ and disease control rate (DCR) of $82 \%$ in 107 patients with FGFR2 fusion or rearrangement. The median follow-up period was 17.8 months and the median PFS and OS were 6.9 and 21.1 months, respectively [32]. Hypophosphatemia, arthralgia, stomatitis, hyponatremia, abdominal pain, and fatigue were the most frequently reported adverse events. This trial has led to the FDA approval of pemigatinib for second line treatment options for patients with FGFR2 fusion or other FGFR2 rearrangement. Pemigatinib is the first approved targeted therapy for BTC. An open-labeled, randomized phase III study (FIGHT-302) was initiated in 2019 to evaluate pemigatinib as first line treatment compared to GemCis (NCT03656536).

Other selective FGFR inhibitors erdafitinib and rogaratinib were evaluated in BTC patients as part of basket trials and there was evidence of antitumor effects [33, 34]. Subsequently, erdafitinib was tested in a phase II study of 14 BTC patients with FGFR alterations. The study resulted in a DCR of $83.3 \%$ with a median PFS of 5.6 months [35]. Additionally, E7090 is a novel, selective FGFR inhibitor of FGFR1, 2, and 3, and is currently under first-in-human clinical evaluation (NCT02275910).

\section{Nonselective inhibitors}

Infigratinib (BGJ398) is a nonselective pan-FGFR inhibitor and showed antitumor activity in a first-in-human phase I basket trial. Three advanced BTC patients (two had FGFR2 fusion and one with an FGFR2 mutation) exhibited stable disease with a 5-20\% reduction in tumor size [36]. In a multi-institutional phase II trial, patients with advanced BTC who had FGFR genetic alterations (e.g., fusion, mutation, and amplification) demonstrated an ORR 
Table 2 Ongoing targeted therapy trials in advanced BTC

\begin{tabular}{|c|c|c|c|c|}
\hline Target & Agent & Phase & Trial ID & Design \\
\hline ATG7 & AbGn-107 & I & NCT02908451 & $\mathrm{BD}$ \\
\hline BRAF & ABM-1310 & I & NCT04190628 & $\mathrm{BD}$ \\
\hline Cancer stemness kinase & BI503 & II & NCT02232633 & $\mathrm{AC}$ \\
\hline CDK4/6 & Abemaciclib & II & NCT03339843 & $\mathrm{AC}$ \\
\hline DNA polymerase & MIV-818 & I/II & NCT03781934 & $\mathrm{AC}$ \\
\hline FGFR & E7090 & II & NCT04238715 & $\mathrm{BD}$ \\
\hline FGFR & Pemigatinib & II & NCT02924376 & $\mathrm{BD}$ \\
\hline FGFR & Pemigatinib & II & NCT04256980 & $\mathrm{BD}$ \\
\hline FGFR & Infigratinib & II & NCT02150967 & $\mathrm{AC}$ \\
\hline FGFR & CPL304110 & I & NCT04149691 & $\mathrm{BD}$ \\
\hline FGFR & Erdafitinib & II & NCT02699606 & $\mathrm{BD}$ \\
\hline FGFR & Infigratinib & II & NCT04233567 & $\mathrm{BD}$ \\
\hline FGFR & TAS-120 & $\mathrm{I} / \mathrm{II}$ & NCT02052778 & $\mathrm{BD}$ \\
\hline FGFR & HMPL-453 & II & NCT04353375 & $\mathrm{BD}$ \\
\hline FGFR2 & Derazantinib & II & NCT03230318 & $\mathrm{BD}$ \\
\hline FGFR4 & INCB062079 & I & NCT03144661 & $\mathrm{BD}$ \\
\hline HER2 & Trastuzumab & II & NCT03613168 & $\mathrm{BD}$ \\
\hline HER2 & A166 & $\mathrm{I} / \mathrm{II}$ & NCT03602079 & BD \\
\hline HER2 & ZW49 & I & NCT03821233 & $\mathrm{BD}$ \\
\hline HER2 & DS-8201a & II & JMA-IIA00423 & $\mathrm{BD}$ \\
\hline IDH1 & AG-120 & III & NCT02989857 & BD \\
\hline IDH1 & IDH305 & I & NCT02381886 & $\mathrm{BD}$ \\
\hline IDH1 & AG-120 & I & NCT02073994 & $\mathrm{BD}$ \\
\hline Multi-kinase & EOC317 & I & NCT03583125 & $\mathrm{BD}$ \\
\hline Notch & CB-103 & $\mathrm{I} / \mathrm{II}$ & NCT03422679 & $\mathrm{AC}$ \\
\hline PARP & Olaparib & II & NCT03212274 & BD \\
\hline PARP & Niraparib & II & NCT03207347 & BD \\
\hline Proteasome & Bortezomib & III & NCT03345303 & $\mathrm{AC}$ \\
\hline TRK & Entrectinib & II & NCT02568267 & BD \\
\hline VEGFR2 & Regorafenib & II & NCT02162914 & $\mathrm{AC}$ \\
\hline VEGFR2 & Apatinib & II & NCT03521219 & $\mathrm{AC}$ \\
\hline VEGFR-2 & Ramucirumab & II & NCT02520141 & $\mathrm{AC}$ \\
\hline ATR/PARP & Ceralasertib/Olaparib & II & NCT03878095 & $\mathrm{BD}$ \\
\hline
\end{tabular}

$A C$ all-comers, $B D$ biomarker driven of $14.8 \%$ and a DCR of $75.4 \%$, respectively. The median PFS was 5.8 months [37]. Hyperphosphatemia, fatigue, stomatitis, and alopecia were the most common adverse events reported. The drug is currently being evaluated in a multicenter, open-label, randomized phase III clinical trial involving patients with advanced BTC harboring FGFR2 gene fusions/translocations in the first line setting in comparison to GemCis (NCT03773302).

TAS-120, ponatinib and derazantibib are nonselective FGFR inhibitors. TAS- 120 exhibited an ORR of $25.0 \%$ and a DCR of $78.6 \%$ in 28 patients with iCCA harboring FGFR2 fusion in a phase I basket study [38]. The phase II study is in progress [39]. In another phase 1 trial with a total of 19 BTC patients, TAS- 120 demonstrated 50\% ORR in patients with FGFR2 fusions and even additional clinical benefit for patients who have progressed on reversible FGFR inhibitors [40]. Ponatinib has shown efficacy in controlling disease in patients with advanced BTC harboring FGFR2 fusion [41]. In a recently completed phase II clinical trial, ponatinib was reported to achieve an ORR of 45\%. The median PFS was 2.4 months and the median OS was 15.7 months (NCT02265341). Another basket trial with ponatinib is still ongoing for advanced solid tumors with an FGFR mutation (NCT02272998). Two of twelve iCCA patients with FGFR genetic alterations demonstrated a partial response in a phase I basket trial with derazantinib [42]. In a phase I/II trial, derazantinib's ORR was $21 \%$, DCR was $82.8 \%$, and it exhibited a median PFS 
5.7 months in 29 advanced iCCA patients with FGFR2 fusion [43]. Derazantinib is currently being tested in a phase II study for advanced iCCA and mixed hepatocellular carcinoma/BTC with FGFR gene aberrance after patients' tumors have progressed with one or more systemic therapies (NCT03230318).

\section{IDH inhibitors}

The incidence of isocitrate dehydrogenase 1/2 (IDH1/ 2) mutations is $4.9-36 \%$ in $\mathrm{iCCA}, 0-7.4 \%$ in eCCA, and $1.5 \%$ in GBC [30]. In general, IDH1 mutations are more common than IDH2 mutations. Pharmacologic inhibitors that are specific to IDH-mutations have been developed [44].

Ivosidenib (AG-120) is an oral, first-in-class inhibitor of mutant IDH1. The treatment efficacy of ivosidenib in BTC was examined in 73 advanced BTC patients with mutated IDH1. Among 72 evaluable patients, four (5\%) patients had a partial response to the treatment. The median PFS and OS were 3.8 months and 13.8 months, respectively [45]. Common adverse events encountered included fatigue, nausea, diarrhea, abdominal pain, decreased appetite, and vomiting. Acquired resistance developed that was associated with a novel IDH2 mutation [46]. The ClarIDHy trial is a global, randomized, double-blind, phase 3 trial to evaluate ivosidenib efficacy in patients with advanced BTC with an IDH1 mutation in comparison to a placebo. In the trial, 185 patients with IDH1 mutated advanced BTC were enrolled and randomized in a 2:1 ratio of ivosidenib to placebo. The median PFS and OS were 2.7 months and 10.8 months with ivosidenib versus 1.4 months and 9.7 months with the placebo. The authors concluded that a favorable trend in OS was seen with ivosidenib treatment [47].

Enasidenib (AG-221) is a selective inhibitor of mutant IDH2 and is currently being assessed in multiple phases I/II clinical trials in subjects with advanced solid tumors, including IDH2 mutated BTC (NCT02273739). Other inhibitors of IDH1 (IDH305, NCT02381886) and panIDH1/2 (AG881, NCT02481154) are currently being tested in patients with BTC in phase I studies.

\section{EGFR inhibitor}

EGFR is overexpressed in $8.1 \%$ of BTC [48], while KRAS mutations in BTCs were identified in $4-40 \%$ of cases [30]. Several EGFR targeted agents have been developed but the antitumor activity observed in BTC was modest. For example, the oral, reversible tyrosine kinase inhibitor (TKI) erlotinib was evaluated in advanced BTC and resulted in three partial responses [49]. When combined with gemcitabine/oxaliplatin (GEMOX) in a phase 3 trial
[50], there was no difference in median OS or PFS; however, a significantly higher ORR was achieved in treatment with chemotherapy plus erlotinib when compared to GEMOX. Furthermore, of 49 evaluable patients treated with the addition of bevacizumab to erlotinib, six patients had partial responses and 25 maintained a stable disease. This combination resulted in a median OS 9.9 months [51]. Although non-randomized phase II trials showed the efficiency of cetuximab for advanced BTC in addition to chemotherapy [52-54], the randomized phase II trial (BINGO) with the combination of cetuximab with GEMOX failed to show OS benefit [20]. Two other randomized phase II trials did not find any considerable benefits of cetuximab and GEMOX or with the addition of capecitabine to GEMOX, even with stratification by KRAS mutation status [55, 56]. Panitumumab was evaluated in a phase II trial in combination with gemcitabine and irinotecan in patients with advanced BTC. The results showed an ORR of $39 \%$ and a median PFS and OS of 9.7 months and 12.9 months, respectively [57]. When panitumumab was combined with GEMOX followed by capcitabine treatment in KRAS wild type irresectable BTC, it showed an ORR of $46 \%$. The median PFS and OS were 6.1 months and 9.5 months, respectively [58].

\section{VEGF inhibitors}

VEGF is highly expressed in $40-75 \%$ of BTC [59]. Several antiangiogenic agents have been developed and tested in clinical trials of BTC; however, VEGF inhibition does not appear to be a relevant therapeutic target in BTC. For example, the combination of bevacizumab with GEMOX in 35 patients with advanced BTC was evaluated in a phase II study and the results showed an ORR of $40 \%$, a median PFS of 7 months, and an OS of 12.7 months [60]. In the other phase II trial involved with a similar regimen, the addition of bevacizumab to GEMOX increased the PFS and but failed to provide an OS benefit [61]. The combination of FOLFIRI with bevacizumab resulted in an ORR of $38.4 \%$ and a median OS of 20 months [62]. Moreover, in a phase II trial to evaluate GemCis with or without cediranib (ABC-03), 124 patients with advanced BTC were enrolled. No significant improvements in either PFS or OS were observed; however, the addition of cediranib did improve the ORR [17]. Single agent sorafenib failed to show any clinical benefit with a reported median OS of 4.4 months [63], and 9 months in patients with advanced BTC [64]. No additional clinical benefit was observed when sorafenib was added to GemCis, yielding a median PFS and OS of 6.5 and 14.4 months, respectively. Furthermore, no significant differences were found when compared to historical controls using GemCis alone [65]. Moreover, a phase II clinical trial with regorafenib as a 
single agent treatment in advanced, refractory BTC exhibited a median PFS and OS was 2.2 and 4.5 months in 43 patients [66]. Sunitinib and Axitinib, were also evaluated in advanced BTC that was refractory to first line treatment [67-69] and vandetanib was evaluated alone or in combination with gemcitabine 70]. However, the role of these agents did not yield promising results.

\section{Tropomyosin receptor kinases (TRKs) inhibitor}

Genomic translocations with chromosomal fusion lead to the constitutive activation of TRKs. Overexpression of TRKs in BTC has been reported but the incidence is variable, ranging from 0 to $20.5 \%$ [71-73]. Larotrectinib is a highly selective TRK inhibitor and was approved for solid tumors with NTRK gene fusion (NCT02122913, NCT02637687 and NCT02576431) [74]. Two patients with BTCs were included in the study, one achieving a nearly $80 \%$ decrease in initial tumor size. Moreover, adverse events were predominantly classified as grade 1 . Entrectinib is a less selective inhibitor of TRK, targeting ROS1 and ALK. It has been evaluated in a phase 1 study of a selected population of solid tumors, including BTCs, and yielded an ORR of 57-86\% [75]. Recent reports from three ongoing phase I/II clinical trials (ALKA-372-001, STARTRK-1, and STARTRK-2) showed an average ORR of 57\% with one partial response in a BTC patient [76]. The most common treatment-related severe adverse events were weight gain, anemia, and neural system disorder.

\section{MET inhibitor}

Overexpression of c-MET has been detected in $12-58 \%$ of iCCA [77, 78] and $16 \%$ of eCCA [77]. TKIs of the MEK pathway have also been studied in this disease. Carbozantinib is a TKI that targets MET and VEGFR2 and has demonstrated limited efficacy in a phase 2 trial, although, one patient with an MET-high tumor received a prolonged clinical benefit from treatment [79]. Tivantinib is an oral, selective c-MET inhibitor and was evaluated in combination with gemcitabine in patients with advanced solid tumors. The results showed that $20 \%$ of 56 patients had a partial response, including one patient with advanced BTC [80]. A non-selective MET inhibitor, merestinib, is currently being evaluated in combination with gemcitabine/cisplatin as a first-line of therapy (NCT02711553). Additionally, the results of a phase 2 trial evaluating Crizotinib, an ALK and c-Met inhibitor, for patients harboring an ALK, MET, or ROS1 alteration are pending completion of the trial (NCT02034981).

\section{MEK and BRAF inhibitors}

The MEK1/2 inhibitor, selumetinib showed an ORR of $12 \%$ among 28 patients with metastatic BTC and median PFS and OS of 3.7 and 9.8 months, respectively [81]. Thirteen patients with advanced BTC were treated with selumetinib in combination with GemCis in a first-line setting (phase $1 \mathrm{~b}$ trial, ABC-04). Patients had three partial responses and 5 maintained stable disease [82]. Selumetinib has continued onto evaluation in a phase 2 study with no results to date (NCT02151084).

Selective oral MEK1/2 inhibitor binimetinib exhibited two partial response and one complete response in BTC [83]. Enhanced antitumor effectiveness of binimetinib was observed in a phase $1 \mathrm{~b}$ study when used in combination with capecitabine in the second-line setting [83]. The median PFS and OS were 3.9 months and 8.0 months, respectively. Additional evaluations of binimetinib in combination with GemCis in phase I/II studies showed an ORR of $36 \%$ in 35 advanced BTC patients with median PFS 6 months and an OS 13.3 months [84]. A study using an identical regimen in advanced BTC is ongoing (NCT02151084). In a randomized phase II study, trametinib monotherapy in patients with advanced BTC failed to show a significant clinical response when compared to the treatment of 5-FU combined with leucovorin or capecitabine [85]. Trametinib was also evaluated in a phase II study of patients with advanced BTC in a second-line setting and showed one partial response among 20 patients and a median PFS of 2.7 months and 1-year survival of $20 \%$ [86]. When combined with pazopanib on 25 patients with advanced BTCs, trametinib showed median PFS of 6.4 months [87].

Mutation of BRAF V600E is rare in BTC with a reported incidence of about 1-5\%, moreover, typically confined to iCCA $[70,88]$. In a phase II basket trial, only one patient out of 12 with iCCA demonstrated a partial response over a year of treatment with vemurafenib [89]. The combination of trametinib and dabrafenib was reported in case studies of advanced BTC with a BRAF V600E mutation and the results showed regression of metastatic lesions [90, 91]. In a basket trial of rare tumors, including BTC harbouring BRAF V600E mutations, treatment with dabrafenib and trametinib resulted in an ORR of $42 \%$ in 35 patients with BTC [92]. The median PFS and OS were 9.2 and 11.7 months, respectively.

\section{HER2/ERBB2 inhibitor}

In BTCs, HER2 overexpression is observed in 5\% of iCCA, $20 \%$ of eCCA, and $19 \%$ of GBC [93]. HER 2 or ERBB2 amplification present in up to $20 \%$ of eCCA but is rare in iCCA $[59,94]$. Alterations of the ERBB family have 
been reported in BTC, comprised of $19 \%$ of $\mathrm{GBC}, 17 \%$ of eCCA [7], and $4.8 \%$ of iCCA cases [93]. Single agent trastuzumab from a retrospective case series showed promising results on GBC but not in iCCA or eCCA with HER2/neu mutation [95]. The addition of chemotherapy to trastuzumab also showed clinical activity in the first line of care for refractory BTC [96]. Trastuzumab is being evaluated with GemCis as second line therapy in HER2-positive BTC in a phase II trial (NCT 03613168) and with GEMOX as a second-line therapy in advanced or recurrent eCCA and GBC (NCT02836847). A phase II trial on trastuzumab emtansine (TDM-1) in bladder cancer, pancreatic adenocarcinoma, and BTC was terminated before full enrollment (NCT02999672). The official results are pending but there was a $14 \%$ overall response of 7 patients from the cohort of pancreatic adenocarcinoma/BTC with median PFS of 2.5 months. Trastuzumab deruxtecan (DS8201) is currently being evaluated in a phase II trial involved with HER2 positive BTC (JMA-IIA00423) [97]. Lapatinib, a dual TKI targeting EGFR and HER2, failed to show antitumor activity in advanced BTC in first and second-line setting as a single agent [98, 99]. An ongoing phase II basket trial (SUMMIT) is examining the efficacy of neratinib (TKI of EGFR, HER2, and HER4) in HER2mutated cancers, including BTCs, and has shown a meaningful clinical response [100]. Interim results indicated an ORR of $10.5 \%$ among 10 enrolled patients with refractory BTC with 2 partial responses and 4 with stable disease. The median PFS was 1.8 months [101]. Varletinib (EGFR/Her2 co-inhibitor) has been evaluated with capecitabine in a randomized, controlled phase II/III trial (TreeTopp) in the second-line setting in BTC (NCT03093870) and with GemCis in first-line setting in advanced BTC (NCT02992340).

\section{PARP inhibitor}

The presence of a germline mutation in BRCA1 or BRCA2 confers an increased lifetime risk of developing BTC [102] and somatic mutations of BRCA1 and BRCA2 have been reported in BTC [6]. BRCA-mutated tumors are often sensitive to poly [ADP-ribose] polymerase (PARP) inhibition [103]. In a retrospective clinical analysis in 18 patients with BRCA-mutated BTC, the median OS for patients, regardless of treatment modality with stage III/IV disease was 25 months, while 40.3 months with stage I/II stage, that appears longer than SEER historical control in general [104]. One of the four patients that received PARP inhibitors obtained a favorable disease response with a PFS duration of 42.6 months and an OS of 64.8 months [104]. A phase II trial of the PARP inhibitor niraparib is planned in patients with advanced-stage malignancies, including BTC, and with known mutations in BAP1 and other DNA double-strand break repair pathway genes (NCT03207347). There are studies of PARP inhibitors, Niraparib and olaparib ongoing in patients with BTC with aberrant gene mutations (NCT04042831, NCT03207347). A basket phase II trial of olaparib for patients with metastatic solid tumors with IDH1 or IDH2 mutations including BTCs has been undertaken (NCT03212274).

Other ongoing trials with molecular based targets in advanced BTC include copanlisib (PI3K inhibitor) (NCT02631590), ABC294640 (sphingosine kinase and JAK/STAT inhibitor) (NCT03377179, NCT03414489), ceritinib and crizotinib (ALK and ROS1 inhibitor) (NCT02374489, NCT02034981) (Table 2).

\section{Immunotherapy}

Over the past two decades cancer treatment has seen much progress for immune-based approaches in solid tumor malignancies. Landmark FDA approvals for various strategies, including immune checkpoint inhibitor (ICI), targeting CTLA4 or the PD1/PDL1 axis have dramatically impacted the lives of patients. Presently, the clinical data on immunotherapy in BTC are limited to small single-arm studies and sub-analyses of basket trials [3, 105], though numerous clinical trials studying ICIs are underway (Tables 3, 4).

\section{Monotherapy}

Pembrolizumab is a highly selective, humanized monoclonal antibody against PD-1 that is designed to block the interaction between PD-1 and its ligands, PD-L1 and PDL2. MMR deficiency has been reported to occur in $5 \%$ to $10 \%$ of CCAs [106]. In a phase II trial of mismatch repair deficient (dMMR) non-colorectal gastrointestinal cancers, 17 patients were treated with pembrolizumab. Of four BTC patients, one had a complete response, one had a partial response, and one stable disease, all with durable responses [107]. In the follow-up study of 8 BTC patients with dMMR, 2 complete responses were achieved and 4 other patients were labeled with stable disease, resulting in an ORR of 25\% [108]. In Keynote158, 22 BTC patients with dMMR and MSI-H were treated with pembrolizumab after progression on or intolerance to at least one line of standard therapy (NCT02628067). The study yielded an ORR of $34.3 \%$ with a median PFS and OS of 4.1 and 23.5 months, respectively. Treatment-related adverse events occurred in 151 patients $(64.8 \%)$. Thirty-four patients (14.6\%) had grade 3 to 5 treatment-related adverse events, therefore, exhibiting a safe profile for pembrolizumab [109]. However, in MSS, MMR proficient BTC, the ORR to ICI monotherapy appears much lower. KEYNOTE-028 (NCT02054806) is an ongoing, multi-cohort, phase Ib trial 
Table 3 Ongoing immunotherapy trials in advanced BTC

\begin{tabular}{|c|c|c|c|c|}
\hline Target & Agent & Phase & Trial ID & Design \\
\hline PD-1 & Toripalimab (JS001) & $\mathrm{I} / \mathrm{II}$ & NCT03867370 & $\mathrm{AC}$ \\
\hline PD-L1 & M7824 & II & NCT03833661 & $\mathrm{AC}$ \\
\hline PD-1 & Pembrolizumab & II & NCT02628067 & $\mathrm{AC}$ \\
\hline PD-L1 & STI-3031 & II & NCT03999658 & $\mathrm{BD}$ \\
\hline CD166 & CX-2009 & $\mathrm{I} / \mathrm{II}$ & NCT03149549 & $\mathrm{AC}$ \\
\hline PD-1/PD-L1 & Pembrolizumab, nivolumab, atezolizumab, ipilimumab & III & NCT04157985 & $\mathrm{AC}$ \\
\hline PD-L1/CTLA-4 & Durvalumab/Tremelimumab & II & NCT04238637 & $\mathrm{AC}$ \\
\hline CTLA-4, PD-1 & Ipilimumab/Nivolumab & II & NCT02834013 & $\mathrm{BD}$ \\
\hline CTLA-4, PD-1 & $\mathrm{XmAb} 20717$ & I & NCT03517488 & $\mathrm{AC}$ \\
\hline CTLA-4, LAG3, PD-1 & XmAb22841। Pembrolizumab & 1 & NCT03849469 & $\mathrm{AC}$ \\
\hline OX40, PD-1 & ABBV-368| ABBV-181 & 1 & NCT03071757 & $\mathrm{AC}$ \\
\hline CD40/CD135 & CDX-1140| CDX-301| Pembrolizumab & 1 & NCT03329950 & $\mathrm{AC}$ \\
\hline TAA & TRK-950 & 1 & NCT02990481 & $\mathrm{AC}$ \\
\hline \multirow[t]{2}{*}{ Vaccine } & Hepcortespenlisimut-L & $\mathrm{I} / \mathrm{II}$ & NCT03042182 & $\mathrm{BD}$ \\
\hline & Hepcortespenlisimut-L & III & NCT02232490 & $\mathrm{BD}$ \\
\hline \multirow[t]{4}{*}{ Cellular } & MUC-1 CART cells & $\mathrm{I} / \mathrm{II}$ & NCT03633773 & $\mathrm{BD}$ \\
\hline & Cytokine-induced killer cells & $\mathrm{I} / \mathrm{II}$ & NCT01868490 & $\mathrm{AC}$ \\
\hline & Tumor Infiltrating Lymphocytes (TIL) & II & NCT03801083 & $\mathrm{AC}$ \\
\hline & Central memory $\mathrm{T}$ cells & II & NCT03820310 & $\mathrm{AC}$ \\
\hline
\end{tabular}

$A C$ all-comers, $B D$ biomarker driven

of pembrolizumab monotherapy for patients with PD-L1positive advanced solid tumors, including PD-L1-positive BTC. Interim safety and efficacy data have been reported for a small cohort of patients with PD-L1-positive BTC. Twenty-four BTC patients with unknown MMR status were enrolled and showed an ORR of $13.0 \%$ with 3 partial responses, and the median PFS and OS were 1.8 and 6.2 months, respectively. The 12-month OS rate was $27.6 \%$ [110]. A larger BTC patient population with MSS is included in the Keynote-158 phase II trial to be treated. Among 104 patients, 6 patients had a confirmed partial response with an ORR of $5.8 \%$. Surprisingly, one patient had a PD-L1 negative tumor. The median duration of response (DOR) was not reached and median PFS and OS were 2.0 and 7.4 months and $50 \%$ of responses were ongoing for at least 24 months [110]. Grade 3-5 treatmentrelated AEs occurred in $13.5 \%$ in Keynote158 (1 case of grade 5 immune-related renal failure) and $16.7 \%$ of patients in Keynote028 (no grade 5). 18.3\% in Keynote158 and $20.8 \%$ of patients in Keynote028 had an immunemediated AE or infusion reaction [110].

Nivolumab is a monoclonal antibody that binds to the PD-1 and blocks its interaction with PD-L1 and PD-L2. The results from a phase II trial in advanced refractory BTC showed an ORR of $11 \%$ with 5 MSS patients out of 46 achieving a partial response and DCR of $50 \%$ with durable responses lasting two years [111]. A phase I study with nivolumab, as monotherapy or combined with chemotherapy in 30 Japanese patients with BTC. One of 30 patients had an objective response in the monotherapy cohort with a median PFS and OS of 1.4 and 5.2 months, respectively [112].

Durvalumab is a human immunoglobulin G1 kappa

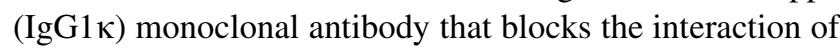
PD-L1 with the PD-1. In this phase I study preliminary results [113], the DCR was $16.7 \%$ at 12 weeks with durvalumab monotherapy. The median duration of response for durvalumab cohort was 9.7 months and median OS was 8.1 months. No unexpected toxicities were observed. Tremelimumab is a monoclonal antibody against CTLA-4. There are ongoing trials exploring the role of tremelimumab in solid tumors, including BTC (NCT01938612).

\section{Other immunotherapies}

T cell based therapy is an emerging field and efficacy has been greatly appreciated in hematological malignancies [114] and melanoma [115]. This therapy for epithelial malignancies, such as BTC is under investigation. A successful case of metastatic BTC treated with mutation specific adoptive T-Cell therapy was reported. The autologous tumor infiltrating lymphocytes (TILs) that were 
Table 4 Ongoing trials of immunotherapy in combination with other in advanced BTC

\begin{tabular}{|c|c|c|c|c|}
\hline Combination & Agent & Phase & Trial ID & Design \\
\hline \multirow[t]{6}{*}{ + Immunotherapy } & Durvalumab/CSF1R inhibitor & II & NCT04301778 & $\mathrm{AC}$ \\
\hline & Anti-PD-1/TC-210 T Cells & $\mathrm{I} / \mathrm{II}$ & NCT03907852 & $\mathrm{BD}$ \\
\hline & Pembrolizumab/Allogeneic NK cell & $\mathrm{I} / \mathrm{II}$ & NCT03937895 & BD \\
\hline & Pembrolizumab/Allogeneic T cells & I & NCT02757391 & $\mathrm{AC}$ \\
\hline & Rovalpituzumab Tesirine/ABBV181 & I & NCT03000257 & $\mathrm{AC}$ \\
\hline & XmAb22841/Pembrolizumab & I & NCT03849469 & $\mathrm{AC}$ \\
\hline+ Immunotherapy $+\mathrm{RT}$ & Autologous DC/Pneumococcal Vaccine/RT & I & NCT03942328 & $\mathrm{AC}$ \\
\hline \multirow[t]{10}{*}{ + Chemotherapy } & TRK950/GemCis & I & NCT03872947 & $\mathrm{AC}$ \\
\hline & Toripalimab/Gemcitabine/5-FU & II & NCT03982680 & $\mathrm{AC}$ \\
\hline & Durvalumab/GemCis & II & NCT04308174 & $\mathrm{AC}$ \\
\hline & Pembrolizumab/CapOx & II & NCT03111732 & $\mathrm{AC}$ \\
\hline & Pembrolizumab/GemCis & III & NCT04003636 & $\mathrm{AC}$ \\
\hline & Durvalumab/Tremelimumab/GemCis & II & NCT03473574 & $\mathrm{AC}$ \\
\hline & Bintrafusp alfa/GemCis & II/III & NCT04066491 & $\mathrm{AC}$ \\
\hline & Pembrolizumab/INT230-6 & $\mathrm{I} / \mathrm{II}$ & NCT03058289 & $\mathrm{AC}$ \\
\hline & Anti-CTLA-4/INT230-6 & $\mathrm{I} / \mathrm{II}$ & NCT03058289 & $\mathrm{AC}$ \\
\hline & Durvalumab/GemCis & III & NCT03875235 & $\mathrm{AC}$ \\
\hline+ Chemotherapy + Targeted therapy & JS001/GEMOX/Lenvatinib & II & NCT03951597 & $\mathrm{AC}$ \\
\hline \multirow[t]{2}{*}{+ Epigenetic modulator } & Durvalumab/Guadecitabine & I & NCT03257761 & $\mathrm{AC}$ \\
\hline & Nivolumab/Entinostat & II & NCT03250273 & $\mathrm{AC}$ \\
\hline \multirow[t]{4}{*}{$+\mathrm{RT}$} & Durvalumab/Tremelimumab/ablation & II & NCT02821754 & $\mathrm{AC}$ \\
\hline & Camrelizumab/Cryoablation & II & NCT04299581 & $\mathrm{AC}$ \\
\hline & Cytokine-induced killer cells/ablation & II/III & NCT02482454 & $\mathrm{AC}$ \\
\hline & Camrelizumab/Radiation & II & NCT03898895 & $\mathrm{AC}$ \\
\hline \multirow[t]{12}{*}{+ Targeted therapy } & Pembrolizumab/Olaparib & II & NCT04306367 & $\mathrm{AC}$ \\
\hline & Atezolizumab/Cobimetinib & II & NCT03201458 & $\mathrm{AC}$ \\
\hline & LMB-100/Tofacitinib & I & NCT04034238 & $\mathrm{AC}$ \\
\hline & Toripalimab/Lenvatinib & II & NCT04211168 & $\mathrm{AC}$ \\
\hline & Durva/AZD6738/Olaparib & II & NCT04298021 & $\mathrm{AC}$ \\
\hline & Durva/AZD6738 & II & NCT04298008 & $\mathrm{AC}$ \\
\hline & Nivo/FT2102 & $\mathrm{I} / \mathrm{II}$ & NCT03684811 & $\mathrm{BD}$ \\
\hline & Durvalumab/Olaparib & II & NCT03991832 & $\mathrm{BD}$ \\
\hline & Nivolumab/TPST-1120 & I & NCT03829436 & $\mathrm{AC}$ \\
\hline & Pembrolizumab/Lenvatinib & II & NCT03895970 & $\mathrm{AC}$ \\
\hline & TQB2450/Anlotinib & $\mathrm{I} / \mathrm{II}$ & NCT03996408 & $\mathrm{AC}$ \\
\hline & Anti-PD-1/IntegrinaV/b8 antagonist & I & NCT04152018 & $\mathrm{AC}$ \\
\hline+ Targeted therapy + RT & Avelumab/Nedisertib/Radiation & $\mathrm{I} / \mathrm{II}$ & NCT04068194 & $\mathrm{AC}$ \\
\hline
\end{tabular}

GemCis gemcitabine cisplatin, GEMOX gemcitabine, oxaliplatin, CapOx capecitabine oxaliplatin, $R T$ radiotherapy, $A C$ all-comers, $B D$ biomarker driven

specific to a mutated antigen ERBB2 interacting protein (ERBB2IP) expressed by the patient's cancer were harvested and expanded in vitro. After lympho-depletive chemotherapy, the patient received the infusion of antigenspecific TILs and there was a significant reduction in the size of tumor lesions [116]. There are several ongoing trials in BTC involved with mesothelin-based immunotoxin LMB-100 (NCT04034238), mesothelin antibody anetumab ravtansine (NCT03102320), and mesothelin CAR-T therapy (NCT03907852).

\section{Combination therapy}

Various studies in combination with different therapy modalities have been evaluated with the goal of improving the efficacy of ICI monotherapy (Table 4). Considering the 
multiple mechanisms adopted by tumor cells to evade the immune system through cancer immunoediting, the combination of ICIs and chemotherapy appears to be a promising strategy. It enhances the recognition and elimination of tumor cells by the host immune system, and reduces the immunosuppressive tumor microenvironment, while eradicating the tumor through DNA damage, inhibiting DNA replication, and preventing mitosis caused by cytotoxic chemotherapy [117]. A similar synergistic effect can be observed when combining ICIs with other treatments such as targeted therapies and radiation due to the immunomodulatory effects of targeted agents and radiation.

A phase I study involved with advanced BTC in Japanese population treated with nivolumab alone or in combination with GemCis showed that 11 of 30 patients had an objective response with a median PFS and OS of 4.2 and 15.4 months in the combination cohort [112]. In a phase I study of advanced BTC treated with the combination of durvalumab plus tremelimumab, preliminary results showed disease control rate was $32.2 \%$ at 12 weeks. The median duration of response was 8.5 months and median OS was 10.1 months [113]. Another phase I study reported the use of tremalimumab in combination with microwave ablation in the patients with advanced refractory BTC [118]. Two patients among sixteen evaluable patients achieved a confirmed partial response. Median PFS and OS was 3.4 and 6.0 months, respectively. An observational study reported the results of the use of lenvatinib plus nivolumab and pembrolizumab in 14 patients with iCCAs. The data showed ORR of $21.4 \%$ with 3 pts achieved partial response (PR) and DCR of 92.9\%. Median PFS was 5.9 months. The most common adverse events included hypertension, aminotransferase elevation and fatigue [119]. Other ongoing trials with ICIs in combination with different regimens in advanced BTC are summarized in Table 4.

\section{Future directions}

The systemic treatment strategies of advanced BTCs have been evolving for the last decade. There is established efficacy of the first-line chemotherapy GemCis and several potential triplet therapy regimens are under the evaluation. Still, no standard second-line chemotherapy regimen has been recommended though efforts have been made to test some candidates. The understanding of the molecular landscape of BTC has shed light on promising precision medicine with targeted therapy. Pemigatinib is approved for treatment of advanced refractory BTC with FGFR2 fusion with other rearrangement. The combination of chemotherapy and targeted agents has not shown enthusiastic outcomes. Nevertheless, several ongoing trials are assessing combination options. Recent breakthroughs of knowledge in cancer immunology have attracted a wave of immunotherapy options being investigated in cancers including BTC. However, the modest efficacy of single agent immunotherapy in advanced BTC is leading to various ongoing clinical trials using combinations of chemotherapy, targeted therapy, radiation therapy and other immunotherapy agents with the purpose of boosting the outcome of single agents. It is unclear the intratumorally immunological effect caused by targeted therapy or radiation therapy in BTC. Nevertheless, immunosuppressive tumor microenvironment composes of different immune cell population (Fig. 1) that needs intelligent design to identify the best therapeutic approaches to improve outcome. The increased numbers of available data from clinical trials concurrently opens more questions to be answered.

Since the patients included in the majority of clinical trials of BTC were heterogenous in terms of origin of diseases, this likely interferes with the interpretation of results from clinical trials no matter which treatment modalities have been applied. Efforts should be taken to enroll patients with specific origin of diseases or specific genetic backgrounds, however, that would certainly require global efforts to recruit enough patients. Fortunately, there are more trials being designed with this consideration, especially in iCCAs and GBC. With these efforts, we should be able to appreciate more significant results from homogenous patient populations and guide clinical practice with a disease location-specific approach. Furthermore, this heterogenicity is not only existing as disease per se, but the genetic background of individual patients is heterogenicity as indicated by mutation landscape and certainly contributes to the heterogenicity of the tumor microenvironment of BTCs as a whole. Furthermore, this plays a critical role in a patient's response to immunotherapy. This heterogenicity has to be considered when the trials are designed, and when data are interpreted.

This notion leads to the other challenge, which is to stratify the subgroup of patients who will be more likely to respond to treatment. For example, there are multiple studies to explore the immune biomarkers at baseline from peripheral blood samples or biopsied tumor samples to stratify the likely responsible patients to immunotherapy, including tumor mutation burden, circulating tumor DNA, tumor infiltrating lymphocytes, PD-L1 expression in the tumor, etc. Currently, it is unclear if these markers would have predicative value in the immunotherapy of BTCs and it remains an important topic to be illuminated.

Lastly, most completed and ongoing trials with the combination strategies are involved with the administration of agents concurrently. It is unclear whether sequential 
Fig. 1 Tumor microenvironment of biliary tract cancer and potential cellular targets in combination with immunotherapy. There are different cell types that compose the immunosuppressive tumor microenvironment. It is a potential strategy to improve the efficacy of immune checkpoint inhibitors in combination with the agents targeting on other immune cells. MDSC myeloid derived suppressive cell, $C A F$ cancer-associated fibroblast, $E C M$ extracellular matrix

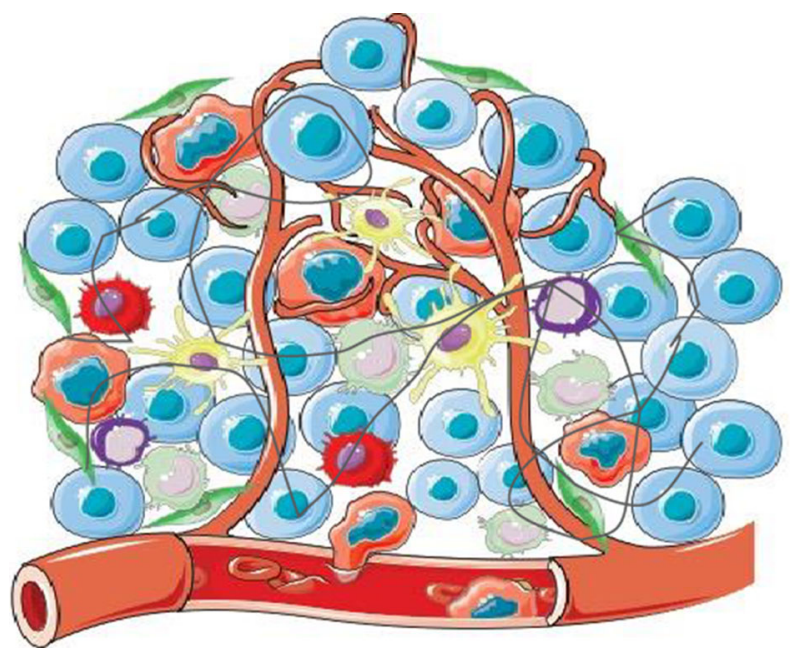

Cancer stem cell

Tumor cell

T cell

Dendric cell

MDSC

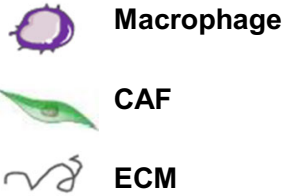

treatment and maintenance therapy would be alternative and superior to concurrent approach. Additional investigation is needed to unravel these possibilities.

Author contributions CX and NM contributed to the collection of information. CX, NM, JF and CM contributed to the writing and editing of this manuscript.

Funding CX is supported by the Physician-Scientist Early Investigator program at National Cancer Institute, National Institute of Health.

\section{Compliance with ethical standards}

Conflicts of interest No conflicts of interest relevant to this manuscript.

Open Access This article is licensed under a Creative Commons Attribution 4.0 International License, which permits use, sharing, adaptation, distribution and reproduction in any medium or format, as long as you give appropriate credit to the original author(s) and the source, provide a link to the Creative Commons licence, and indicate if changes were made. The images or other third party material in this article are included in the article's Creative Commons licence, unless indicated otherwise in a credit line to the material. If material is not included in the article's Creative Commons licence and your intended use is not permitted by statutory regulation or exceeds the permitted use, you will need to obtain permission directly from the copyright holder. To view a copy of this licence, visit http://creativecommons. org/licenses/by/4.0/.

\section{References}

1. Razumilava N, Gores GJ. Cholangiocarcinoma. Lancet. 2014;383:2168-79.

2. Rizvi S, Gores GJ. Pathogenesis, diagnosis, and management of cholangiocarcinoma. Gastroenterology. 2013;145:1215-29.

3. Rizvi S, Khan SA, Hallemeier CL, et al. Cholangiocarcinoma evolving concepts and therapeutic strategies. Nat Rev Clin Oncol. 2018;15:95-111.
4. Wongjarupong N, Assavapongpaiboon B, Susantitaphong $\mathrm{P}$, et al. Non-alcoholic fatty liver disease as a risk factor for cholangiocarcinoma: a systematic review and meta-analysis. BMC Gastroenterol. 2017;17:149.

5. Sheth S, Bedford A, Chopra S. Primary gallbladder cancer: recognition of risk factors and the role of prophylactic cholecystectomy. Am J Gastroenterol. 2000;95:1402-10.

6. Nakamura H, Arai Y, Totoki Y, et al. Genomic spectra of biliary tract cancer. Nat Genet. 2015;47:1003-10.

7. Churi CR, Shroff R, Wang Y, et al. Mutation profiling in cholangiocarcinoma: prognostic and therapeutic implications. PLoS ONE. 2014;9:e115383.

8. Khan SA, Tavolari S, Brandi G. Cholangiocarcinoma: epidemiology and risk factors. Liver Int. 2019;39(Suppl 1):19-31.

9. Mukkamalla SKR, Naseri HM, Kim BM, et al. Trends in incidence and factors affecting survival of patients with cholangiocarcinoma in the United States. J Natl Compr Canc Netw. 2018;16:370-6.

10. Bertuccio P, Malvezzi M, Carioli G, et al. Global trends in mortality from intrahepatic and extrahepatic cholangiocarcinoma. J Hepatol. 2019;71:104-14.

11. Siegel RL, Miller KD, Jemal A. Cancer statistics, 2020. CA Cancer J Clin. 2020;70:7-30.

12. Hang $\mathrm{H}$, Jeong $\mathrm{S}$, Sha $\mathrm{M}$, et al. Cholangiocarcinoma: anatomical location-dependent clinical, prognostic, and genetic disparities. Ann Transl Med. 2019;7:744.

13. Mavros MN, Economopoulos KP, Alexiou VG, et al. Treatment and prognosis for patients with intrahepatic cholangiocarcinoma: systematic review and meta-analysis. JAMA Surg. 2014;149:565-74.

14. Ducreux M, Van Cutsem E, Van Laethem JL, et al. A randomised phase II trial of weekly high-dose 5-fluorouracil with and without folinic acid and cisplatin in patients with advanced biliary tract carcinoma: results of the 40955 EORTC trial. Eur J Cancer. 2005;41:398-403.

15. Kornek GV, Schuell B, Laengle F, et al. Mitomycin C in combination with capecitabine or biweekly high-dose gemcitabine in patients with advanced biliary tract cancer: a randomised phase II trial. Ann Oncol. 2004;15:478-83.

16. Okusaka T, Nakachi K, Fukutomi A, et al. Gemcitabine alone or in combination with cisplatin in patients with biliary tract cancer: a comparative multicentre study in Japan. $\mathrm{Br} \mathrm{J}$ Cancer. 2010;103:469-74.

17. Valle JW, Wasan H, Lopes A, et al. Cediranib or placebo in combination with cisplatin and gemcitabine chemotherapy for 
patients with advanced biliary tract cancer (ABC-03): a randomised phase 2 trial. Lancet Oncol. 2015;16:967-78.

18. Valle J, Wasan H, Palmer DH, et al. Cisplatin plus gemcitabine versus gemcitabine for biliary tract cancer. $\mathrm{N}$ Engl $\mathrm{J}$ Med. 2010;362:1273-81.

19. Valle JW, Bai L-Y, Orlova R, et al. Ramucirumab (RAM) or merestinib (MER) or placebo (PL) plus gemcitabine (GEM) and cisplatin (CIS) as first-line treatment for advanced or metastatic biliary tract cancer (BTC): A randomized, double-blind, phase II study. J Clin Oncol 2020;38:1.

20. Malka D, Cervera P, Foulon S, et al. Gemcitabine and oxaliplatin with or without cetuximab in advanced biliary-tract cancer (BINGO): a randomised, open-label, non-comparative phase 2 trial. Lancet Oncol. 2014;15:819-28.

21. Moehler M, Maderer A, Ehrlich A, et al. Safety and efficacy of afatinib as add-on to standard therapy of gemcitabine/cisplatin in chemotherapy-naive patients with advanced biliary tract cancer: an open-label, phase I trial with an extensive biomarker program. BMC Cancer. 2019;19:55.

22. Morizane C, Okusaka T, Mizusawa J, et al. Combination gemcitabine plus S-1 versus gemcitabine plus cisplatin for advanced/ recurrent biliary tract cancer: the FUGA-BT (JCOG1113) randomized phase III clinical trial. Ann Oncol. 2019;30:1950-8.

23. Rao S, Cunningham D, Hawkins RE, et al. Phase III study of 5FU, etoposide and leucovorin (FELV) compared to epirubicin, cisplatin and 5FU (ECF) in previously untreated patients with advanced biliary cancer. Br J Cancer. 2005;92:1650-4.

24. Yamashita Y, Taketomi A, Itoh S, et al. Phase II trial of gemcitabine combined with 5-fluorouracil and cisplatin (GFP) chemotherapy in patients with advanced biliary tree cancers. Jpn J Clin Oncol. 2010;40:24-8.

25. Kanai M, Hatano E, Kobayashi S, et al. A multi-institution phase II study of gemcitabine/cisplatin/S-1 (GCS) combination chemotherapy for patients with advanced biliary tract cancer (KHBO 1002). Cancer Chemother Pharmacol. 2015;75:293-300.

26. Sakai D, Kanai M, Kobayashi S, et al. Randomized phase III study of gemcitabine, cisplatin plus S-1 (GCS) versus gemcitabine, cisplatin (GC) for advanced biliary tract cancer (KHBO1401-MITSUBA). Ann Oncol. 2018;29:205.

27. Shroff RT, Javle MM, Xiao L, et al. Gemcitabine, cisplatin, and nab-paclitaxel for the treatment of advanced biliary tract cancers: a phase 2 clinical trial. JAMA Oncol. 2019;5:824-30.

28. Lamarca A, Hubner RA, David Ryder W, et al. Second-line chemotherapy in advanced biliary cancer: a systematic review. Ann Oncol. 2014;25:2328-38.

29. Lamarca A, Palmer DH, Wasan HS, et al. ABC-06 I A randomised phase III, multi-centre, open-label study of active symptom control (ASC) alone or ASC with oxaliplatin/5-FU chemotherapy (ASC + mFOLFOX) for patients (pts) with locally advanced/metastatic biliary tract cancers $(\mathrm{ABC})$ previously-treated with cisplatin/gemcitabine (CisGem) chemotherapy. J Clin Oncol. 2019;37:1.

30. Valle JW, Lamarca A, Goyal L, et al. New horizons for precision medicine in biliary tract cancers. Cancer Discov. 2017;7:943-62.

31. Ikeda M, Maruki Y, Ueno M, et al. Frequency and clinicopathological characteristics of biliary tract carcinomas harboring the FGFR2-fusion gene: a prospective observational study (PRELUDE study). Ann Oncol. 2019;30:mdz247.

32. Abou-Alfa GK, Sahai V, Hollebecque A, et al. Pemigatinib for previously treated, locally advanced or metastatic cholangiocarcinoma: a multicentre, open-label, phase 2 study. Lancet Oncol. 2020;21(5):671-84.

33. Nishina T, Takahashi S, Iwasawa R, et al. Safety, pharmacokinetic, and pharmacodynamics of erdafitinib, a pan-fibroblast growth factor receptor (FGFR) tyrosine kinase inhibitor, in patients with advanced or refractory solid tumors. Invest New Drugs. 2018;36:424-34.

34. Schuler M, Cho BC, Sayehli CM, et al. Rogaratinib in patients with advanced cancers selected by FGFR mRNA expression: a phase 1 dose-escalation and dose-expansion study. Lancet Oncol. 2019;20:1454-66.

35. Park JO, Feng Y-H, Chen Y-Y, et al. Updated results of a phase IIa study to evaluate the clinical efficacy and safety of erdafitinib in Asian advanced cholangiocarcinoma (CCA) patients with FGFR alterations. J Clin Oncol. 2019;37:4117.

36. Nogova L, Sequist LV, Perez Garcia JM, et al. Evaluation of BGJ398, a fibroblast growth factor receptor 1-3 kinase inhibitor, in patients with advanced solid tumors harboring genetic alterations in fibroblast growth factor receptors: results of a global phase I, dose-escalation and dose-expansion study. J Clin Oncol. 2017;35:157-65.

37. Javle M, Lowery M, Shroff RT, et al. Phase II study of BGJ398 in patients with FGFR-altered advanced cholangiocarcinoma. J Clin Oncol. 2018;36:276-82.

38. Meric-Bernstam F, Arkenau H, Tran B, et al. O-001-efficacy of TAS-120, an irreversible fibroblast growth factor receptor (FGFR) inhibitor, in cholangiocarcinoma patients with FGFR pathway alterations who were previously treated with chemotherapy and other FGFR inhibitors. Ann Oncol. 2018;29:v100.

39. Hollebecque A, Doi T, Saavedra O, et al. A phase II study of futibatinib (TAS-120) in patients (pts) with advanced (adv) solid tumors harboring fibroblast growth factor receptor (FGFR) genomic aberrations. J Clin Oncol. 2020;38:TPS470.

40. Goyal L, Arkenau H-T, Tran B, et al. O-020-early clinical efficacy of TAS-120, a covalently bound FGFR inhibitor, in patients with cholangiocarcinoma. Ann Oncol. 2017;28:145.

41. Borad MJ, Champion MD, Egan JB, et al. Integrated genomic characterization reveals novel, therapeutically relevant drug targets in FGFR and EGFR pathways in sporadic intrahepatic cholangiocarcinoma. PLoS Genet. 2014;10:e1004135.

42. Papadopoulos KP, El-Rayes BF, Tolcher AW, et al. A Phase 1 study of ARQ 087, an oral pan-FGFR inhibitor in patients with advanced solid tumours. Br J Cancer. 2017;117:1592-9.

43. Mazzaferro V, El-Rayes BF, Droz Dit Busset M, et al. Derazantinib (ARQ 087) in advanced or inoperable FGFR2 gene fusion-positive intrahepatic cholangiocarcinoma. $\mathrm{Br} \mathrm{J}$ Cancer. 2019;120:165-71.

44. Golub D, Iyengar N, Dogra S, et al. Mutant isocitrate dehydrogenase inhibitors as targeted cancer therapeutics. Front Oncol. 2019;9:417.

45. Lowery MA, Burris HA, Janku F, et al. Safety and activity of ivosidenib in patients with IDH1-mutant advanced cholangiocarcinoma: a phase 1 study. Lancet Gastroenterol Hepatol. 2019;4:711-20.

46. Harding JJ, Lowery MA, Shih AH, et al. Isoform switching as a mechanism of acquired resistance to mutant isocitrate dehydrogenase inhibition. Cancer Discov. 2018;8:1540-7.

47. Abou-Alfa GK, Macarulla T, Javle MM, et al. Ivosidenib in IDH1-mutant, chemotherapy-refractory cholangiocarcinoma (ClarIDHy): a multicentre, randomised, double-blind, placebocontrolled, phase 3 study. Lancet Oncol. 2020;21:796-807.

48. Nakazawa K, Dobashi Y, Suzuki S, et al. Amplification and overexpression of c-erbB-2, epidermal growth factor receptor, and c-met in biliary tract cancers. J Pathol. 2005;206:356-65.

49. Philip PA, Mahoney MR, Allmer C, et al. Phase II study of erlotinib in patients with advanced biliary cancer. J Clin Oncol. 2006;24:3069-74.

50. Lee J, Park SH, Chang HM, et al. Gemcitabine and oxaliplatin with or without erlotinib in advanced biliary-tract cancer: a 
multicentre, open-label, randomised, phase 3 study. Lancet Oncol. 2012;13:181-8.

51. Lubner SJ, Mahoney MR, Kolesar JL, et al. Report of a multicenter phase II trial testing a combination of biweekly bevacizumab and daily erlotinib in patients with unresectable biliary cancer: a phase II Consortium study. J Clin Oncol. 2010;28:3491-7.

52. Gruenberger B, Schueller J, Heubrandtner U, et al. Cetuximab, gemcitabine, and oxaliplatin in patients with unresectable advanced or metastatic biliary tract cancer: a phase 2 study. Lancet Oncol. 2010;11:1142-8.

53. Rubovszky G, Lang I, Ganofszky E, et al. Cetuximab, gemcitabine and capecitabine in patients with inoperable biliary tract cancer: a phase 2 study. Eur J Cancer. 2013;49:3806-12.

54. Borbath I, Ceratti A, Verslype C, et al. Combination of gemcitabine and cetuximab in patients with advanced cholangiocarcinoma: a phase II study of the Belgian Group of Digestive Oncology. Ann Oncol. 2013;24:2824-9.

55. Chen JS, Hsu C, Chiang NJ, et al. A KRAS mutation statusstratified randomized phase II trial of gemcitabine and oxaliplatin alone or in combination with cetuximab in advanced biliary tract cancer. Ann Oncol. 2015;26:943-9.

56. Ole Larsen F, Taksony Solyom Hoegdall D, Hoegdall E, et al. Gemcitabine, capecitabine and oxaliplatin with or without cetuximab in advanced biliary tract carcinoma. Acta Oncol. 2016;55:382-5.

57. Sohal DP, Mykulowycz K, Uehara T, et al. A phase II trial of gemcitabine, irinotecan and panitumumab in advanced cholangiocarcinoma. Ann Oncol. 2013;24:3061-5.

58. Leone F, Marino D, Cereda S, et al. Panitumumab in combination with gemcitabine and oxaliplatin does not prolong survival in wild-type KRAS advanced biliary tract cancer: a randomized phase 2 trial (Vecti-BIL study). Cancer. 2016;122:574-81.

59. Yoshikawa D, Ojima H, Iwasaki M, et al. Clinicopathological and prognostic significance of EGFR, VEGF, and HER2 expression in cholangiocarcinoma. $\mathrm{Br} \mathrm{J}$ Cancer. 2008;98:418-25.

60. Zhu AX, Meyerhardt JA, Blaszkowsky LS, et al. Efficacy and safety of gemcitabine, oxaliplatin, and bevacizumab in advanced biliary-tract cancers and correlation of changes in 18-fluorodeoxyglucose PET with clinical outcome: a phase 2 study. Lancet Oncol. 2010;11:48-54.

61. Brechon M, Dior M, Dreanic J, et al. Addition of an antiangiogenic therapy, bevacizumab, to gemcitabine plus oxaliplatin improves survival in advanced biliary tract cancers. Invest New Drugs. 2018;36:156-62.

62. Guion-Dusserre JF, Lorgis V, Vincent J, et al. FOLFIRI plus bevacizumab as a second-line therapy for metastatic intrahepatic cholangiocarcinoma. World J Gastroenterol. 2015;21:2096-101.

63. Bengala C, Bertolini F, Malavasi N, et al. Sorafenib in patients with advanced biliary tract carcinoma: a phase II trial. Br J Cancer. 2010;102:68-72.

64. El-Khoueiry AB, Rankin CJ, Ben-Josef E, et al. SWOG 0514: a phase II study of sorafenib in patients with unresectable or metastatic gallbladder carcinoma and cholangiocarcinoma. Invest New Drugs. 2012;30:1646-51.

65. Lee JK, Capanu M, O'Reilly EM, et al. A phase II study of gemcitabine and cisplatin plus sorafenib in patients with advanced biliary adenocarcinomas. $\mathrm{Br} \mathrm{J}$ Cancer. 2013;109:915-9.

66. Sun W, Patel A, Normolle D, et al. A phase 2 trial of regorafenib as a single agent in patients with chemotherapy-refractory, advanced, and metastatic biliary tract adenocarcinoma. Cancer. 2019;125:902-9.
67. Neuzillet C, Seitz J-F, Fartoux L, et al. Sunitinib as second-line treatment in patients with advanced intrahepatic cholangiocarcinoma (SUN-CK phase II trial): safety, efficacy, and updated translational results. J Clin Oncol. 2015;33:1.

68. Okano N, Ueno M, Morizane C, et al. Multicenter phase II trial of axitinib monotherapy for advanced biliary tract cancer refractory to gemcitabine-based chemotherapy. Ann Oncol. 2019;30:1.

69. Yi JH, Thongprasert S, Lee J, et al. A phase II study of sunitinib as a second-line treatment in advanced biliary tract carcinoma: a multicentre, multinational study. Eur J Cancer. 2012;48:196-201.

70. Santoro A, Gebbia V, Pressiani T, et al. A randomized, multicenter, phase II study of vandetanib monotherapy versus vandetanib in combination with gemcitabine versus gemcitabine plus placebo in subjects with advanced biliary tract cancer: the VanGogh study. Ann Oncol. 2015;26:542-7.

71. Hsiao SJ, Zehir A, Sireci AN, et al. Detection of tumor NTRK gene fusions to identify patients who may benefit from tyrosine kinase (TRK) inhibitor therapy. J Mol Diagn. 2019;21:553-71.

72. Westphalen CB, Preinfalk A, Kruger S, et al. Neurotrophic tropomyosin receptor kinase (NTRK) and nerve growth factor (NGF) are not expressed in Caucasian patients with biliary tract cancers: pooled data from three independent cohorts. Clin Transl Oncol. 2019;21:1108-11.

73. Yang XQ, Xu YF, Guo S, et al. Clinical significance of nerve growth factor and tropomyosin-receptor-kinase signaling pathway in intrahepatic cholangiocarcinoma. World J Gastroenterol. 2014;20:4076-84.

74. Drilon A, Laetsch TW, Kummar S, et al. Efficacy of larotrectinib in trk fusion-positive cancers in adults and children. N Engl J Med. 2018;378:731-9.

75. Drilon A, Siena S, Ou SI, et al. Safety and antitumor activity of the multitargeted pan-TRK, ROS1, and ALK Inhibitor entrectinib: combined results from two phase I trials (ALKA-372-001 and STARTRK-1). Cancer Discov. 2017;7:400-9.

76. Doebele RC, Drilon A, Paz-Ares L, et al. Entrectinib in patients with advanced or metastatic NTRK fusion-positive solid tumours: integrated analysis of three phase 1-2 trials. Lancet Oncol. 2020;21:271-82.

77. Terada T, Nakanuma Y, Sirica AE. Immunohistochemical demonstration of MET overexpression in human intrahepatic cholangiocarcinoma and in hepatolithiasis. Hum Pathol. 1998;29:175-80.

78. Miyamoto M, Ojima H, Iwasaki M, et al. Prognostic significance of overexpression of c-Met oncoprotein in cholangiocarcinoma. Br J Cancer. 2011;105:131-8.

79. Goyal L, Zheng H, Yurgelun MB, et al. A phase 2 and biomarker study of cabozantinib in patients with advanced cholangiocarcinoma. Cancer. 2017;123:1979-88.

80. Pant S, Saleh M, Bendell J, et al. A phase I dose escalation study of oral c-MET inhibitor tivantinib (ARQ 197) in combination with gemcitabine in patients with solid tumors. Ann Oncol. 2014;25:1416-21.

81. Bekaii-Saab T, Phelps MA, Li X, et al. Multi-institutional phase II study of selumetinib in patients with metastatic biliary cancers. J Clin Oncol. 2011;29:2357-63.

82. Bridgewater J, Lopes A, Beare S, et al. A phase $1 \mathrm{~b}$ study of Selumetinib in combination with Cisplatin and Gemcitabine in advanced or metastatic biliary tract cancer: the ABC-04 study. BMC Cancer. 2016;16:153.

83. Bendell JC, Javle M, Bekaii-Saab TS, et al. A phase 1 doseescalation and expansion study of binimetinib (MEK162), a potent and selective oral MEK1/2 inhibitor. $\mathrm{Br} \mathrm{J}$ Cancer. 2017;116:575-83. 
84. Lowery MA, Bradley M, Chou JF, et al. Binimetinib plus gemcitabine and cisplatin phase $\mathrm{i} / \mathrm{ii}$ trial in patients with advanced biliary cancers. Clin Cancer Res. 2019;25:937-45.

85. Kim RD, McDonough SL, El-Khoueiry AB, et al. SWOG S1310: randomized phase II trial of single agent MEK inhibitor trametinib vs. 5-fluorouracil or capecitabine in refractory advanced biliary cancer. J Clin Oncol. 2017;35:4016.

86. Ikeda M, Ioka T, Fukutomi A, et al. Efficacy and safety of trametinib in Japanese patients with advanced biliary tract cancers refractory to gemcitabine. Cancer Sci. 2018;109:215-24.

87. Shroff RT, Yarchoan M, O'Connor A, et al. The oral VEGF receptor tyrosine kinase inhibitor pazopanib in combination with the MEK inhibitor trametinib in advanced cholangiocarcinoma. Br J Cancer. 2017;116:1402-7.

88. Goeppert B, Frauenschuh L, Renner M, et al. BRAF V600Especific immunohistochemistry reveals low mutation rates in biliary tract cancer and restriction to intrahepatic cholangiocarcinoma. Mod Pathol. 2014;27:1028-34.

89. Hyman DM, Puzanov I, Subbiah V, et al. Vemurafenib in Multiple Nonmelanoma Cancers with BRAF V600 Mutations. N Engl J Med. 2015;373:726-36.

90. Kocsis J, Arokszallasi A, Andras C, et al. Combined dabrafenib and trametinib treatment in a case of chemotherapy-refractory extrahepatic BRAF V600E mutant cholangiocarcinoma: dramatic clinical and radiological response with a confusing synchronic new liver lesion. J Gastrointest Oncol. 2017;8:E32-8.

91. Lavingia V, Fakih M. Impressive response to dual BRAF and MEK inhibition in patients with BRAF mutant intrahepatic cholangiocarcinoma-2 case reports and a brief review. J Gastrointest Oncol. 2016;7:E98-102.

92. Wainberg ZA, Lassen UN, Elez E, et al. Efficacy and safety of dabrafenib (D) and trametinib (T) in patients (pts) with BRAF V600E-mutated biliary tract cancer (BTC): a cohort of the ROAR basket trial. J Clin Oncol. 2019;37:187.

93. Galdy S, Lamarca A, McNamara MG, et al. HER2/HER3 pathway in biliary tract malignancies; systematic review and meta-analysis: a potential therapeutic target? Cancer Metastasis Rev. 2017;36:141-57.

94. Kim HJ, Yoo TW, Park DI, et al. Gene amplification and protein overexpression of HER-2/neu in human extrahepatic cholangiocarcinoma as detected by chromogenic in situ hybridization and immunohistochemistry: its prognostic implication in nodepositive patients. Ann Oncol. 2007;18:892-7.

95. Javle M, Churi C, Kang HC, et al. HER2/neu-directed therapy for biliary tract cancer. J Hematol Oncol. 2015;8:58.

96. Nam AR, Kim JW, Cha Y, et al. Therapeutic implication of HER2 in advanced biliary tract cancer. Oncotarget. 2016;7:58007-21.

97. Ohba A, Morizane C, Ueno M, et al. Multicenter phase II study of trastuzumab deruxtecan (DS-8201) for HER2-positive unresectable or recurrent biliary tract cancer: HERB trial. J Clin Oncol. 2020;38:TPS4654.

98. Peck J, Wei L, Zalupski M, et al. HER2/neu may not be an interesting target in biliary cancers: results of an early phase II study with lapatinib. Oncology. 2012;82:175-9.

99. Ramanathan RK, Belani CP, Singh DA, et al. A phase II study of lapatinib in patients with advanced biliary tree and hepatocellular cancer. Cancer Chemother Pharmacol. 2009;64:777-83.

100. Hyman DM, Piha-Paul SA, Won H, et al. HER kinase inhibition in patients with HER2- and HER3-mutant cancers. Nature. 2018;554:189-94.

101. Harding J, Cleary J, Shapiro G, et al. O-005-treating HER2mutant advanced biliary tract cancer with neratinib: benefits of HER2-directed targeted therapy in the phase 2 SUMMIT 'basket' trial. Ann Oncol. 2019; https://doi.org/10.1093/annonc/ mdz154.004

102. Cancer risks in BRCA2 mutation carriers. J Natl Cancer Inst 1999; 91: 1310-1316.

103. Bryant HE, Schultz N, Thomas HD, et al. Specific killing of BRCA2-deficient tumours with inhibitors of poly(ADP-ribose) polymerase. Nature. 2005;434:913-7.

104. Golan T, Raitses-Gurevich M, Kelley RK, et al. Overall survival and clinical characteristics of brca-associated cholangiocarcinoma: a multicenter retrospective study. Oncologist. 2017;22:804-10.

105. Kelley RK, Bridgewater J, Gores GJ, et al. Systemic therapies for intrahepatic cholangiocarcinoma. J Hepatol. 2020;72:353-63.

106. Silva VW, Askan G, Daniel TD, et al. Biliary carcinomas: pathology and the role of DNA mismatch repair deficiency. Chin Clin Oncol. 2016;5:62.

107. Le DT, Uram JN, Wang H, et al. PD-1 blockade in tumors with mismatch-repair deficiency. N Engl J Med. 2015;372:2509-20.

108. Le DT, Durham JN, Smith KN, et al. Mismatch repair deficiency predicts response of solid tumors to PD-1 blockade. Science. 2017;357:409-13.

109. Marabelle A, Le DT, Ascierto PA, et al. Efficacy of pembrolizumab in patients with noncolorectal high microsatellite instability/mismatch repair-deficient cancer: results from the phase II KEYNOTE-158 study. J Clin Oncol. 2020;38:1-10.

110. Bang Y-J, Ueno M, Malka D, et al. Pembrolizumab (pembro) for advanced biliary adenocarcinoma: results from the KEYNOTE028 (KN028) and KEYNOTE-158 (KN158) basket studies. J Clin Oncol. 2019;37:1

111. Kim RD, Chung V, Alese OB, et al. A phase 2 multi-institutional study of nivolumab for patients with advanced refractory biliary tract cancer. JAMA Oncol. 2020;6(6):1-8.

112. Ueno $M$, Ikeda $M$, Morizane $C$, et al. Nivolumab alone or in combination with cisplatin plus gemcitabine in Japanese patients with unresectable or recurrent biliary tract cancer: a non-randomised, multicentre, open-label, phase 1 study. Lancet Gastroenterol Hepatol. 2019;4:611-21.

113. Ioka T, Ueno M, Oh D-Y, et al. Evaluation of safety and tolerability of durvalumab (D) with or without tremelimumab (T) in patients (pts) with biliary tract cancer (BTC). J Clin Oncol. 2019;37:1

114. Park JH, Riviere I, Gonen M, et al. Long-term follow-up of CD19 CAR therapy in acute lymphoblastic leukemia. N Engl J Med. 2018;378:449-59.

115. Dudley ME, Yang JC, Sherry R, et al. Adoptive cell therapy for patients with metastatic melanoma: evaluation of intensive myeloablative chemoradiation preparative regimens. J Clin Oncol. 2008;26:5233-9.

116. Tran E, Turcotte S, Gros A, et al. Cancer immunotherapy based on mutation-specific CD4 $+\mathrm{T}$ cells in a patient with epithelial cancer. Science. 2014;344:641-5.

117. Yan Y, Kumar AB, Finnes H, et al. Combining immune checkpoint inhibitors with conventional cancer therapy. Front Immunol. 2018;9:1739.

118. Xie C, Duffy AG, Mabry-Hrones D, et al. Tremelimumab in combination with microwave ablation in patients with refractory biliary tract cancer. Hepatology. 2019;69:2048-60.

119. Lin J, Shi W, Zhao S, et al. Lenvatinib plus checkpoint inhibitors in patients (pts) with advanced intrahepatic cholangiocarcinoma (ICC): preliminary data and correlation with nextgeneration sequencing. J Clin Oncol. 2018;36:500.

Publisher's Note Springer Nature remains neutral with regard to jurisdictional claims in published maps and institutional affiliations. 